\title{
Gaia and the use of White Dwarfs as Advanced Physics laboratories
}

\author{
Jordi Isern* \\ Institut de Ciéncies de l'Espai (CSIC), Spain \\ Institut d'Estudis Espacials de Catalunya (IEEC), Spain \\ Reial Acadèmia de Ciències $i$ Arts de Barcelona (RACAB), Spain \\ E-mail: iserneice.cat
}

\begin{abstract}
White dwarfs are the final remnants of low and intermediate mass stars and their evolution is essentially a long lasting process of cooling. The tool that allows to compare the theoretical models with the observations is their luminosity function, that is, the number of stars per unit volume and luminosity interval. The shape of the bright branch of this function is only sensitive to the average cooling rate and, thus, it is possible to use it to check for the possible existence of additional non standard sources or sinks of energy able to modify the expected 'normal evolution'. Despite the recent improvements introduced by cosmological surveys like the SDSS and the SCSS catalogues, one of the main difficulties to achieve this goal is still the size of the samples. Gaia, launched in 2013, will allow to obtain, in a next future, the fundamental properties of $\sim 400,000$ white dwarfs. Such a sample will be statistically significant and will allow the detection of small deviations from the normal cooling process. As an example, we describe here the case of axions, a not yet detected weakly interacting particle introduced to solve the so called CP-problem of the Standard Model of particles. In particular, we show that their inclusion noticeably improves the agreement between the theoretical and observational white dwarf luminosity functions, thus providing a first hint that axions could exist. This improvement is not only valid for the luminosity function obtained with all three catalogues but also for the luminosity function of the galactic thin and thick disks, suggesting that the change of the luminosity function shape is due to an intrinsic property of white dwarfs and not to a fluctuation of the star formation rate. The best fit is obtained for axion masses around $6 \mathrm{meV}$, and values larger than $16 \mathrm{meV}$ could probably be excluded.
\end{abstract}

The Golden Age of Cataclysmic Variables and Related Objects - III

7-12 September 2015

Palermo, Italy

\footnotetext{
*Speaker.
} 


\section{Introduction}

White dwarfs are the final remnants of low and intermediate mass stars. They are supported by the pressure of their degenerate electrons and their evolution from the planetary nebula phase to its disappearance is essentially a long lasting process of cooling. They can be imagined as a degenerate core that contains the bulk of the mass and acts as an energy reservoir, surrounded by a semi degenerate envelope that controls the flux of energy from the interior to the free space. The main source of energy is the gravothermal readjustment of the star, complemented with the latent heat and gravitation sedimentation upon crystallization, and with the gravitational diffusion of heavy chemical species like ${ }^{22} \mathrm{Ne}$. An additional term, that strongly depends on the size of the outer layers, is the residual burning of hydrogen in the envelope. The sinks of energy are thermal neutrinos, that freely escape, and photons that diffuse through the envelope. A detailed discussion can be found in $[1,2,3,4,5]$.

The luminosity strongly depends on the properties of the envelope (mass, chemical composition and structure) as well as on the total mass and radius of the white dwarf. The main characteristics of the envelope is its tendency to become stratified, the lightest elements tending to be placed on top of the heaviest ones as a consequence of the strong gravitational field. However, this behavior is counterbalanced by convection, molecular diffusion and other processes that tend to restore the chemical homogeneity. In any case, the $\sim 80 \%$ of white dwarfs shows the presence of $\mathrm{H}$-lines in their spectra while the remaining $\sim 20 \%$ not. This proportion is not constant along the cooling sequence. The first ones are called DAs and the second ones non-DAs. The most common interpretation is that the DAs have a double layered envelope made of $\mathrm{H}\left(M_{\mathrm{H}} \sim 10^{-4} M_{\mathrm{WD}}\right)$ and $\mathrm{He}\left(M_{\mathrm{He}} \sim 10^{-2} M_{\odot}\right)$ while the non-DAs have just a single He layer or an extremely thin $\mathrm{H}$ layer. An additional complication is that the initial conditions at the moment of formation are not well known and for the moment it is not possible to disentangle which part of this behavior is inherited and which part is evolutive [5].

The simplicity of the structure and evolution of white dwarfs have converted them into useful tools to constrain new ideas in Physics. This has been the case of axions, monopoles, or the secular variation of the gravitational constant, just to cite few examples. However, thanks to the fundamental data that are being obtained by Gaia, the precision of the future catalogues will be much better than the existing ones and this will allow to use white dwarfs as laboratories for advanced physics. The purpose of this talk is to show, using the axion case, how white dwarfs can be used to obtain new insight.

\section{The luminosity function}

The luminosity function (LF) is defined as the number density of white dwarfs of a given luminosity per unit magnitude interval:

$$
n(l)=\int_{M_{1}}^{M_{\mathrm{u}}} \Phi(M) \Psi(t) \tau_{\mathrm{cool}}(l, M) d M
$$

where $t$ satisfies the condition $t=T-t_{\text {cool }}(l, M)-t_{\mathrm{PS}}(M)$ and $l=-\log \left(L / L_{\odot}\right), M$ is the mass of the parent star (for convenience all white dwarfs are labeled with the mass of the main sequence 
progenitor), $t_{\text {cool }}$ is the cooling time down to luminosity $l, \tau_{\text {cool }}=d t / d M_{\mathrm{bol}}$ is the characteristic cooling time, $t_{\mathrm{PS}}$ is the lifetime of the progenitor of the white dwarf, and $T$ is the age of the population under study. $M_{\mathrm{u}}$ and $M_{\mathrm{l}}$ are the maximum and the minimum masses of the main sequence stars able to produce a white dwarf of luminosity $l$. Therefore, $M_{1}$ satisfies the condition

$$
T=t_{\text {cool }}\left(l, M_{1}\right)+t_{\mathrm{PS}}\left(M_{1}\right)
$$

The remaining quantities, the initial mass function, $\Phi(M)$, and the star formation rate, $\Psi(t)$, are not known a priori and depend on the properties of the stellar population under study. In order to compare theory with observations and since the total density of white dwarfs is not well known yet, the computed luminosity function is usually normalized to the bin with the smallest error bar, traditionally the one with $l \simeq 3$.

Eq. (2.1) contains three sets of terms, the observational ones, $n$, the stellar ones, $t_{\mathrm{cool}}, \tau_{\mathrm{cool}}, t_{\mathrm{PS}}$, $M_{\mathrm{u}}$ and $M_{\mathrm{l}}$, and the galactic ones, $\Phi$, and $\Psi$. Therefore, if the stellar properties are well known it is possible to obtain information about the galactic ones and viceversa.

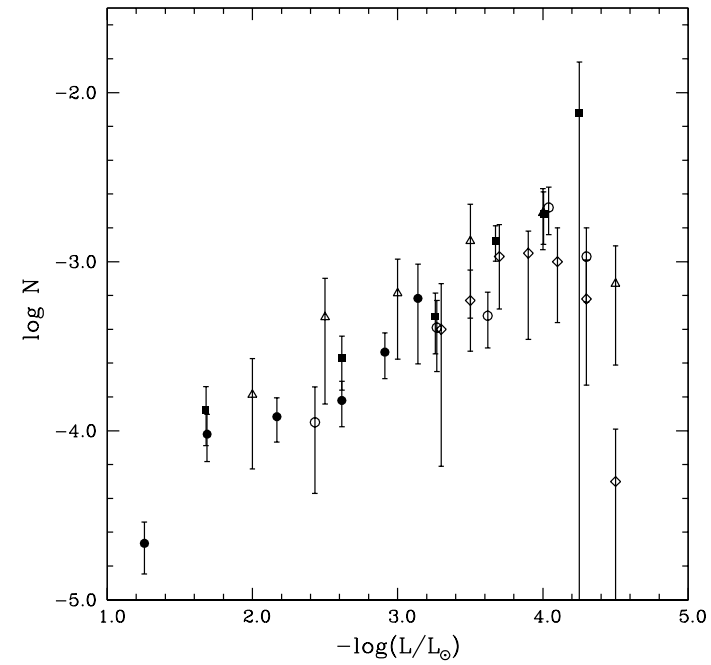

Figure 1: Luminosity functions obtained before the large cosmological surveys - [7], full circles; [8], full squares; [9], open triangles; [10], open diamonds; [11], open circles.

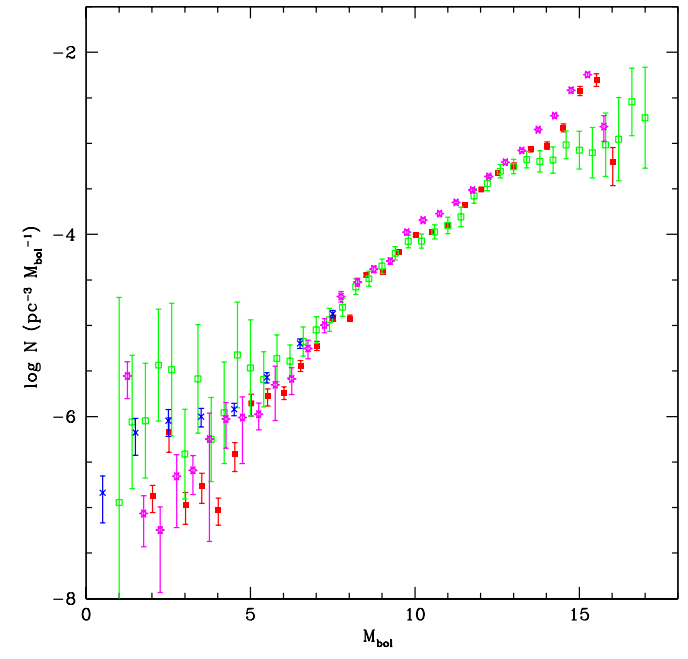

Figure 2: Luminosity functions obtained from the SDSS catalogue. Blue [12] and red [13] contain DA and non-DA white dwarfs. The green one [14] only contains DAs. Purple [15] points were obtained from the SCSS catalogue. They contain DA and non-DA stars.

The first luminosity function was derived four decades ago by [6] and since then it has been noticeably improved with the work of many authors (Fig. 1 and 2). The monotonic behavior of this function clearly proves that the evolution of white dwarfs is just a cooling process. The sharp cut-off at low luminosities is a consequence of the finite age of the Galaxy.

Figure 1 displays the LF obtained before the advent of the large cosmological surveys. The main problem of these functions is the small number of stars in the sample. The Sloan Digital Sky Survey (SDSS) has noticeably improved the accuracy of the new LFs. Figure 2 displays two LFs obtained from this catalogue. One, red points [13], was built with 6,000 stars identified with the photometry and the USNO-B proper motion catalogue. The other, green points [14] contains 3528 
spectroscopically identified DA stars. The discrepancies between both functions at low luminosities are well understood and can be attributed to the methods used to obtain the effective temperature and gravity. Furthermore, in this region it is difficult to separate DAs from non-DAs. At high luminosities, $M_{\text {bol }} \lesssim 6$, the dispersion of both LFs is very large. The reason is that the method of reduced proper motions to identify white dwarfs is not appropriate for bright stars. The blue points [12] display the luminosity function obtained with the UV technique, which in turn is not appropriate for dim stars and becomes rapidly inefficient for $M_{\mathrm{bol}} \gtrsim 7$. Since they overlap it is possible to extend the LF to high luminosities just assuming continuity.

One of the problems is that in the SDSS the integration time is fixed and the S/N depends on the brightness of the source and this can introduce systematic errors. Fortunately, the LF, purple points [15], obtained from the analysis of the proper motions in the Super COSMOS Sky Survey (SCSS) provides values that not deviate from the SDSS ones.

Besides the limited size of the samples, there are many uncertainties associate to fundamental parameters like luminosity, effective temperature, distance and masses of white dwarfs. Fortunately, this situation will change in a nearby future. Gaia is an ESA mission that was launched on December 19th 2013. Its goal is to measure the fundamental properties of a billion of stars. It is expected that among them there will be of the order of 300,000 to 500,000 white dwarfs. An amount large enough to build a catalogue that will be complete up to a distance of $50 \mathrm{pc}$ and half complete up to a distance of $300 \mathrm{pc}$. Unfortunately the spectral resolution is not good enough to derive the mass and effective temperature, and a parallel program from the ground will be necessary to obtain the detailed spectra of such stars.

An important property of Eq. (2.1) is that the bright branch of the luminosity function is only sensitive to the average characteristic cooling time of white dwarfs at the corresponding luminosity when this function is normalized. The reason [16] is that since $\tau_{\text {cool }}$ is not strongly dependent of the mass of the star it can be substituted by a convenient average and removed from the integral leading to

$$
n(l)=\left\langle\tau_{\mathrm{cool}}\right\rangle \int_{M_{\mathrm{l}}}^{M_{\mathrm{u}}} \phi(M) \psi\left(T-t_{\mathrm{cool}}-t_{\mathrm{ps}}\right) d M .
$$

This integral contains the initial mass function, that has not changed very much with time, and the star formation rate that is not known and could have been very irregular along the lifetime of the galaxy. However, since the stellar populations are dominated by low mass stars, which have very long lifetimes, it turns out that old, low mass stars are still producing white dwarfs, and every bin of the luminosity function contains white dwarfs produced by stars that were born at very different epochs. Bright bins mean short cooling times and according to eq. 2.2 small $M_{1}$ values. But since the lifetime of stars increases very sharply when the mass decreases, the lower limit of the integral in eq. (2.3) is almost independent of the luminosity, and the value of the integral is absorbed by the normalization constant. The final outcome is that the bright branch of the luminosity function is only sensitive to the averaged characteristic cooling time of white dwarfs, $n(l) \propto\left\langle\tau_{\text {cool }}\right\rangle$.

Concerning the stellar terms of eq. 2.1, the properties of parent stars are reasonably well known and all the stellar codes provide similar results. The main uncertainties are precisely in the AGB phase and affect the chemical composition of the white dwarf and the relationship between the masses of the white dwarf and its progenitor. 
Globally, the evolution of the luminosity of white dwarfs can be written as:

$$
L+L_{V}+L_{\mathrm{x}}=-\int_{0}^{M_{\mathrm{WD}}} C_{\mathrm{v}} \frac{d T}{d t} d m-\int_{0}^{M_{\mathrm{WD}}} T\left(\frac{\partial P}{\partial T}\right)_{V, X_{0}} \frac{d V}{d t} d m+\left(l_{\mathrm{s}}+e_{\mathrm{s}}\right) \dot{M}_{\mathrm{s}}+\varepsilon_{\mathrm{x}}
$$

where the 1.h.s. of the equation represents the sinks of energy due to photons, neutrinos and any exotic particle, while the r.h.s. contains the sources of energy, the heat capacity of the star, the work due to the change of volume, the contribution of the latent heat and gravitational settling upon crystallization, times the rate of crystallization, $\dot{M}_{\mathrm{S}}[4]$, and any additional exotic source. This equation has to be complemented with a relationship connecting the temperature of the core with the luminosity of the star. Typically $L \propto T_{\mathrm{c}}^{\alpha}$, where $\alpha \simeq 2.7$.

The cooling process can be roughly divided in four stages: neutrino cooling, fluid cooling, crystallization and Debye cooling. When white dwarfs are very hot and bright, $\log \left(L / L_{\odot}\right) \gtrsim-1$ or $M_{\text {bol }} \lesssim 8$, the cooling is dominated by the neutrino emission. This stage is very complicated because of its dependence on the initial conditions of the star, and on the complex and not yet well understood behavior of the envelope. Fortunately, since neutrino emission is dominant, the different thermal structures converge to a unique one when $\log \left(L / L_{\odot}\right) \lesssim-1.5$ (Fig. 4). The time necessary to reach this value is very short, $\lesssim 10^{8}$ years $[1,3]$.

When the luminosity is in the range $-1.5 \lesssim \log \left(L / L_{\odot}\right) \lesssim-3$, or $8.5 \lesssim M_{\text {bol }} \lesssim 12.5$, the main source of energy is gravothermal and the plasma behaves as a fluid, with properties reasonably well known [17]. The flux of energy through the envelope is controlled by a thick non degenerate layer with an opacity dominated by hydrogen (if present) and helium since metals are almost absent due to the efficient gravitational diffusion. The main source of uncertainty is the chemical structure of the interior, which depends on the adopted rate of the ${ }^{12} C(\alpha, \gamma){ }^{16} O$ reaction and on the treatment of semi-convection and overshooting. If this rate is high, the oxygen abundance is higher in the center than in the outer layers resulting in a reduction of the specific heat and a change in the solidification temperature [18].

Crystallization starts around $\log \left(L / L_{\odot}\right) \sim-3$, or $M_{\text {bol }} \sim 8.5$, although it depends on the mass and detailed chemical composition. It introduces two new sources of energy: latent heat and sedimentation. In the case of Coulomb plasmas, the latent heat is small, of the order of $k_{\mathrm{B}} T_{\mathrm{s}}$ per nuclei, where $k_{\mathrm{B}}$ is the Boltzmann constant and $T_{\mathrm{S}}$ is the temperature of solidification. Its contribution to the total luminosity is small, $\sim 5 \%$, but not negligible. During crystallization, the equilibrium chemical compositions of the solid and liquid plasmas are not equal. If the solid is denser it sinks towards the central region. If it is lighter, it rises upwards and melts when the liquefaction temperature, which depends on the density, is reached. The net effect is a migration of heavier elements towards the central regions with the subsequent release of gravitational energy. The efficiency of the process depends on the detailed chemical and it is maximum for a mixture made of half oxygen and half carbon uniformly distributed through all the star.

When almost all the star has solidified, the specific heat follows the Debye's law and the star quickly fades. However, the outer layers still have very large temperatures as compared with the Debye's one, and since their total heat capacity is still large enough, they prevent the sudden disappearance of the white dwarf in the case, at least, of thick envelopes [3].

The evolution models adopted here are the BaSTI models [19]. All the existing models predict the same temporal evolution of the luminosity before crystallization. Only BASTI and La Plata 


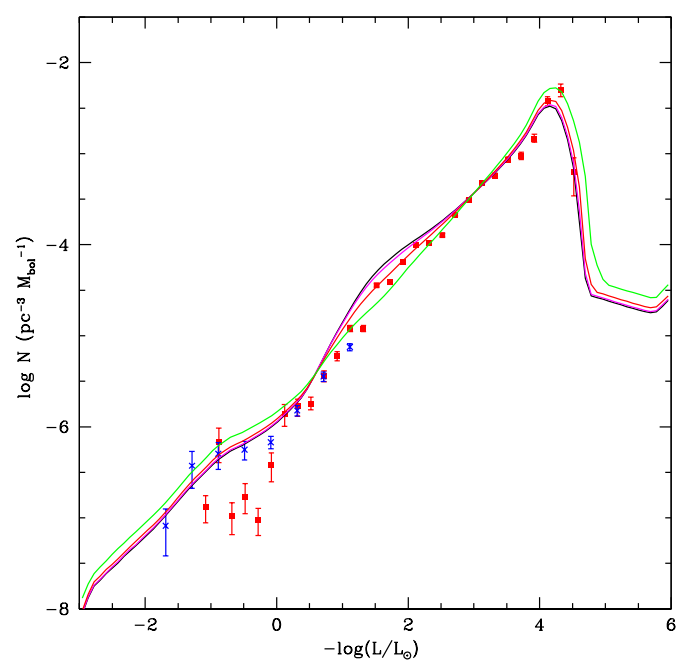

Figure 3: Luminosity function of DA and non-DA white dwarfs obtained from the SDSS catalogue [13, 12] compared with the theoretical values (black line). Color lines have been obtained assuming that axions exist (DFSZ type) and that they couple with electrons with different strengths. The black, magenta, red and green lines represent the LFs obtained for $g_{a e} \times 10^{13}=0.0,1.12$, 2.24 and 4.48 (see text).

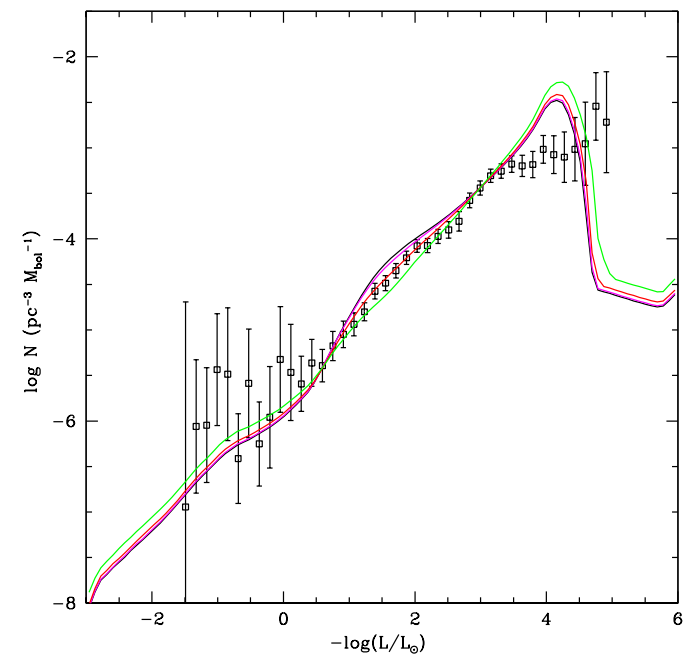

Figure 4: Luminosity function of DA white dwarfs from the SDSS catalogue [14]. Continuous lines have the same meaning as in Fig. 3.

models have fully included sedimentation associated to solidification and both essentially coincide. Furthermore, when the energy release associated to the diffusion of ${ }^{22} \mathrm{Ne}$ is included, the La Plata models can reconcile the age of white dwarfs and the age obtained from the turning point in NGC6791 [20]. The initial final mass relationship adopted here is the empirical one obtained by [21]. Its influence is not critical as far as the production of white dwarfs by stars with masses smaller than solar is not strongly suppressed.

Figures 3,4,5 and 6 display he theoretical LF using BaSTI models and assuming a constant star formation rate. In all cases, if the brightest and dimmest parts are excluded, there is a reasonable agreement between theoretical and observational values. This agreement is maintained if different but smooth star formation rates are used. Only irregularities with ages smaller than 1 to 2 Gyr, those that have no time to affect the normalization point, can modify the shape of the LF.

All these theoretical LFs have a feature in common, they predict an excess of white dwarfs in the region of $M_{\mathrm{bol}} \sim 10$. If all the uncertainties are considered, this feature is not significant except for the fact that is present in all the luminosity functions, that is associated with region where photons overwhelm neutrinos (Fig. 7), and it is present in the LF of the thin and thick galactic disks and even in that of the halo (not represented here due to the poor quality of the observed LF).

\section{The axion case}

One of the long standing problems of the Standard Model is the so called 'strong CP problem', that is, the existence in the Lagrangian of the quantum chromodynamics of a term that violates the 


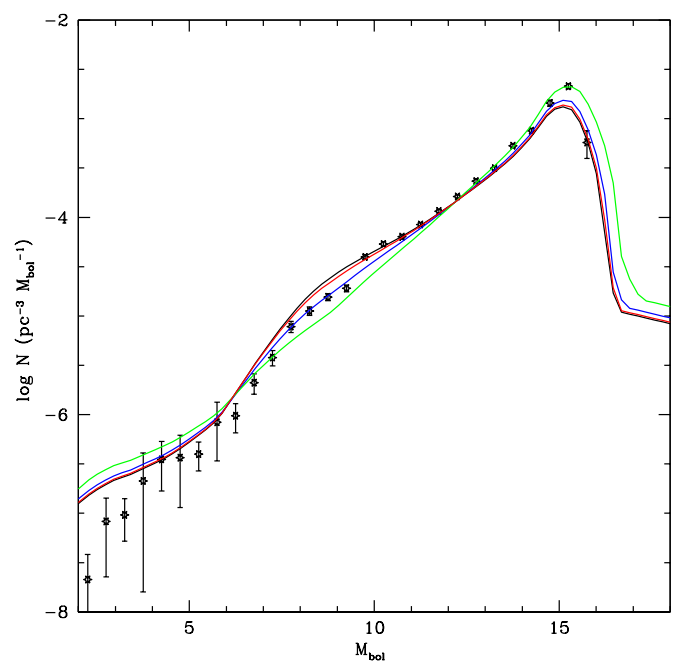

Figure 5: Luminosity function of $\mathrm{DA}$ and no-nDA white dwarfs obtained from the SCSS catalogue [15]. The black, blue, green and red lines represent the LFs obtained for $g_{a e} \times 10^{13}=0.0,1.12,2.24$ and 4.48 (see text).

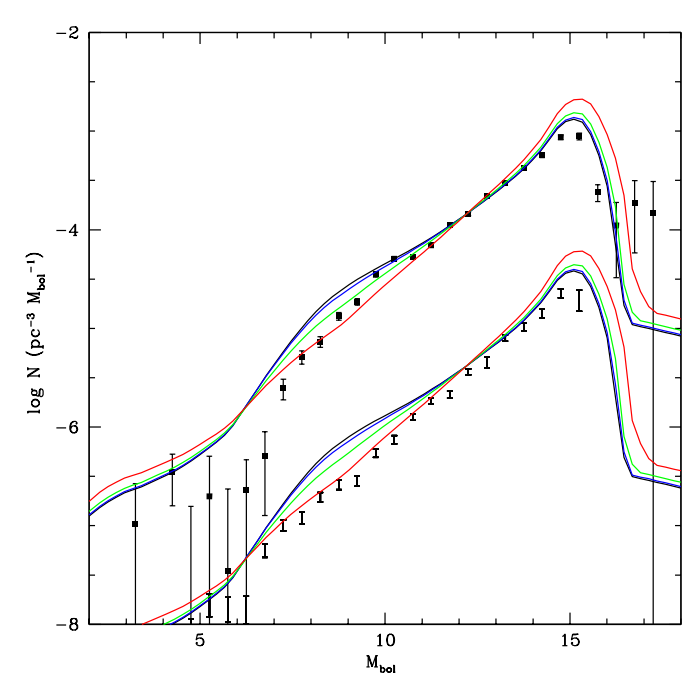

Figure 6: Luminosity function of $\mathrm{DA}$ and no-nDA white dwarfs from the thin (up) and thick (down) disks, obtained from the SCSS catalogue [15]. Continuous lines have the same meaning as in Fig. 3.

charge-parity symmetry and is not observed in Nature. To solve this problem, Roberto Peccei and Helen Quinn introduced a new symmetry that brokens at energies of the order of $f_{\mathrm{a}} \sim 10^{9}$ $10^{12} \mathrm{GeV}$. This symmetry gives raise to a new particle, the axion, a boson, that has a mass $m_{\mathrm{a}}=$ $6 \mathrm{meV}\left(10^{9} \mathrm{GeV} / f_{\mathrm{a}}\right)$, not fixed by theory. The larger is the mass, the larger is the interaction with matter [22].

The interaction with photons and fermions can be described as:

$$
L_{a}=-\frac{1}{4} g_{a \gamma} F_{\mu v} \tilde{F}^{\mu v}-\sum_{\text {fermions }} g_{a i} a \overline{\psi_{i}} \gamma_{5} \psi_{i}
$$

where $g_{a \gamma}\left(\mathrm{GeV}^{-1}\right)$ and $g_{a i}$ are the coupling constants, $F$ is the electromagnetic field tensor and $\gamma_{5}$ is the corresponding Dirac field. The values that these constants take are model dependent. Here, the so called DFSZ model of axions is adopted since it predicts a profuse production of axions in the hot and dense interior of white dwarfs as a consequence of the interaction with the electrons. For white dwarfs in the range $8.5 \lesssim M_{\text {bol }} \lesssim 12.5$, the production of neutrinos and axions is dominated by bremsstrahlung. The specific energy loss rates are $\varepsilon_{v} \propto T^{8}$ and $\varepsilon_{a} \propto T^{4}$ respectively, which means that when neutrino emission disappears axions are still active. Fig.7 shows that this happens just in the region where current theoretical models predict an excess of white dwarfs.

Figures 3,4,5 and 6 also show the luminosity functions obtained when axions are included for different values of $g_{a e}$. Since $g_{a e}=2.8 \times 10^{-14} \cos ^{2} \beta m_{\mathrm{a}}[\mathrm{meV}]$, where $\beta$ is a free parameter, the corresponding mass of axions are $0,4,8$ and $16 \mathrm{meV}$ respectively assuming $\cos \beta=1$. Figures show that the agreement with observations noticeably improves if it is assumed that axions exist and have masses of the order of $6 \mathrm{meV}$, that is if $f_{\mathrm{a}} \sim 10^{9} \mathrm{GeV}$. Unfortunately, the present uncertainties 


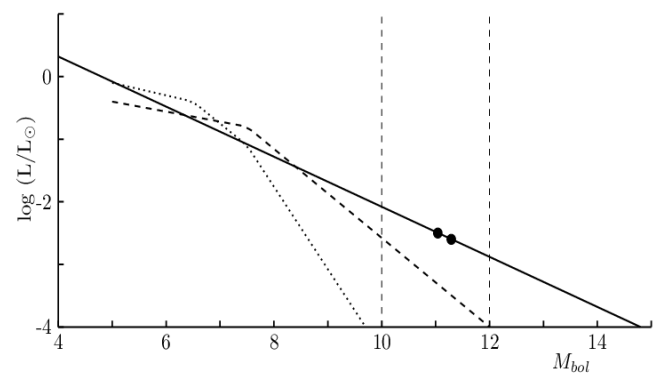

Figure 7: Approximate evolution of the energy sinks as a $0.6 M_{\odot}$ white dwarf fades away. The continuous line represents the photon luminosity, the dotted line represents the neutrino luminosity and the dashed line the axion luminosity assuming a mass of $6 \mathrm{meV}$. Dots display the position of R548 and G117.

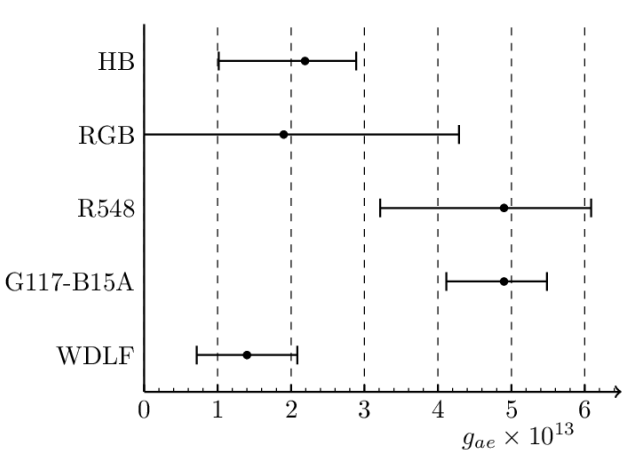

Figure 8: Coupling constants of the axion-electron interaction necessary to account for the observed properties of several objects (HB [26], RGB [27], R548 [29], G117-B15A [28] and WDLF this paper).

do not allow to go further than to provide a hint. Similar results are obtained using the La Plata models [23].

\section{Discussion and conclusions}

At first glance, this value of the mass of the axion, few $m e V$, could be considered a bit strange or even irrelevant. In fact, this was one of the arguments that were used when it was proposed [24], for the first time, the existence of DFSZ axions with a mass of $\sim 8.5 \mathrm{meV}$ to account for the anomalous behavior of G117-B15A, a variable white dwarf. However, nowadays there are strong arguments to believe that particles with sub-eV masses constitute a new frontier in Physics [25]. In any case, the existence of such axions should have important consequences in Astrophysics and Cosmology.

From the astrophysical point of view, the axion emission, like the neutrino one, modifies the evolution of stars and introduces subtle changes in their 'standard' behavior. In this sense, the expected improvement in the precision of the fundamental parameters of stars that Gaia will provide will be critical to detect such anomalies.

Besides the effects found in the white dwarf LF, several anomalies have also been found in the variables G117-B15A and R548, in the ratio between the number of stars in the horizontal branch (HB), and in the red giant branch, and in the maximum luminosity of the red giants (RGB), see Fig. 8. No one of these cases has enough precision to claim the detection of axions, but taken together they show a clear concordance [30,31].

The case of variable white dwarfs deserve some comments. Depending on their location in the HR diagram and their atmospheric composition they are called pulsating PG1159 or GW Vir stars, DBV or V777 stars, and DAV or ZZ Ceti stars (see [5] for a review). As the star cools down, the Brunt-Wäisälä frequency decreases and pulsations gradually shift to lower frequencies. At the same time, since the star contracts, the radius decreases and the frequency tends to increase. In the 
DAV case the temperature is so low that the last effect is negligible and the change of the period of pulsation can be directly related to the change of the central temperature of the star, $\dot{\Pi} / \Pi \propto-\dot{T} / T$, thus allowing to test the cooling theory of white dwarfs. Furthermore, these variables are placed in a region where the neutrino emission has already stopped and the influence of axions, if they exist is more clear (Fig.8).

G117-B15A is a ZZ Ceti star that was discovered by McGraw \& Robinson in 1976 and has been monitored since then. The last measured value of the secular drift of its main period of pulsation $(\Pi \sim 215 \mathrm{~s})$ is $\dot{\Pi}=4.07 \pm 0.61 \times 10^{-15} \mathrm{~s} / \mathrm{s}$ after correcting for the proper motion [32]. This drift has also been measured for the $213 \mathrm{~s}$ period of R548, the ZZ Ceti itself [33]. It amounts $\dot{\Pi} \approx 1.3 \times 10^{-15} \mathrm{~s} / \mathrm{s}$ and is in complete agreement with the values found in G117-B15A. This drift is very small and it has only been possible to measure it in two white dwarfs since it is necessary to organize runs of twenty four hours of coordinated observations during time baselines of 30 years to avoid alliases and to obtain the required precision.

The asteroseismological analysis of both stars indicates that the cooling rate is larger than expected if the pulsation modes are trapped in the outer envelope [28, 29]. This poses a problem since the trapping process strongly depends on the detailed chemical gradients. The chemical structure of these layers is built during the AGB phase and strongly depends on the methods used to treat convection and pulses during this epoch. An important uncertainty comes from the ${ }^{22} \mathrm{Ne}$ profile, the third most abundant isotope in the star, which is usually neglected in calculations. Other problems come from the fact that these outer regions are partially degenerated and not all the physical inputs, specially the axion emissivities, are correctly computed at this regime.

Two of the most important problems in Cosmology is to determine the nature of dark energy and dark matter. Dark energy represents $\sim 70 \%$ of the energy content of the Universe and its density is of the order of $\varepsilon \sim 5 \mathrm{GeVm}^{-3}$, or in natural units $\sim \mathrm{meV}^{4}$. One of the more natural assumptions is to identify it with the energy of the vacuum, which is interpreted as the sum of the fundamental states of all the quantum fields, taking into account that bosons contribute positively and fermions negatively, and that this sum has to be bound at some energy scale. If the Planck mass is adopted as a natural energy cut off, the density of energy that is obtained is $10^{121} \mathrm{GeVm}^{-3}$, a really huge quantity. If the question were at which energy the cut-off should be placed to reproduce the density of the Universe, the answer would be $\sim 10 \mathrm{meV}$, just the region where the mass of the axion is placed, and the one that in the past was considered irrelevant. Notice that now it is also known that neutrinos have their mass in this region.

Dark matter represents $\sim 25 \%$ of the energy of the Universe. Axions have always been a natural candidate. If its mass were of the order of $10 \mu \mathrm{eV}\left(f_{\mathrm{a}} \sim 10^{12} \mathrm{GeV}\right)$ they could by themselves explain the nature of dark matter. If its mass were bigger, it should be necessary to invoke a mixture of WIMPS and axions. In any case, the contribution of axions depends on the moment, before or after inflation, at which the symmetry is broken and, according to some models, axions with a mass of few meV $\left(f_{\mathrm{a}} \sim 10^{9} \mathrm{GeV}\right)$ could account for the major part of dark matter [35, 36], although some fine tuning would be necessary in some cases.

Of course these arguments do not prove neither that axions exist, nor that they have a DFSZ nature with a mass of few meV. They just show that these effects, astrophysical and cosmological, show some concordance. If the results exposed here were correct, probably IAXO (International AXion Observatory) could directly detect the solar axions and settle the question. 
Anyway, future precise surveys, similar to those that Gaia will allow, will convert white dwarfs into a useful tool to examine many other problems of modern Physics

\section{Acknowledgments}

This work has been supported by the MINECO-FEDER grants ESP2013-47637-P \& ESP201341268-R, and the grant 2014SGR1458 of the Generalitat de Catalunya.

\section{References}

[1] Iben, I., Tutukov, A. V. Cooling of low-mass carbon-oxygen dwarfs from the planetary nucleus stage through the crystallization stage ApJ 282 (1984) 615

[2] Koester, D., Schoenberner, D. Evolution of white dwarfs A\&A 154 (1986) 125

[3] D’Antona, F., Mazzitelli, I. The fastest evolving white dwarfs ApJ 347 (1989) 934

[4] Isern, J., García-Berro, E., Hernanz, M., Mochkovitch, R., The physics of white dwarfs J. Phys. Cond. Mat. 10 (1998) 11263

[5] Althaus, L. G., Córsico, A. H., Isern, J., García-Berro, E., Evolutionary and pulsational properties of white dwarf stars AAR $\mathbf{1 8}$ (2010) 471

[6] Weidemann, V. White Dwarfs ARA\&A 6 (1968) 351

[7] Liebert, J., Dahn, C.C., Monet, D.G. The luminosity function of white dwarfs ApJ 332 (1988) 891

[8] Evans, D.W. The APM proper motion project. I- High proper motion stars MNRAS 255 (1992) 521

[9] Oswalt, T.D., Smith, J.A., Wood, M.A., Hintzen, P. A lower lif; mit of 9.5 Gyr on the age of the galactic disk from the oldest white dwarf stars Nature 382 (1996) 692

[10] Legget, S.K., Ruiz,M.T., Bergeron, P. The cool white dwarf luminosity function and the age of the galactic disk ApJ 497 (1998) 294

[11] Knox, R.A. Hawkins, M.R.S., Hambly, N.C. A survey for cool white dwarfs and the age of the galactic disk MNRAS 306 (199) 736

[12] Krzesinski, J., et al. A hot white dwarf luminosity function from the Sloan Digital Sky Survey A\&A $\mathbf{5 0 8}$ (2009) 339

[13] Harris, H. C., et al. The white dwarf luminosity function from Sloan Digital Sky Survey imaging data AJ 131 (2006) 571

[14] DeGennaro, S., et al. White dwarf luminosity and mass functions from Sloan Digital Sky Survey spectra AJ 135 (2008) 1

[15] Rowell, N., Hambly, N.C. White dwarfs in the Super Cosmos Sky Survey: the thin disk, thick disk and spheroid luminosity funcions MNRAS 417 (2011) 93

[16] Isern, J., García-Berro, E. White dwarfs as physics laboratories: the axion case MemSAI 79 (2008) 545

[17] Segretain, L., et al. Cooling theory of crystallized white dwarfs ApJ 434 (1994) 652

[18] Salaris, M., et al. The Cooling of CO White Dwarfs: Influence of the Internal Chemical Distribution ApJ 486 (1997) 413 
[19] Salaris, M., et al. A large stellar evolution data base for population synthesis studies. VI. White dwarf cooling sequences ApJ 716 (2010) 1241

[20] García-Berro, E., et al. The white dwarf cooling age of NGC 6791 Nature 465 (2010) 194

[21] Catalan, S., et al. The initial-final mass relationship of white dwarfs revisited: effect on the luminosity function and mass distribution MNRAS 387 (2008) 1693

[22] Raffelt, G.G. Stars as laboratories for fundamental physics: the astrophysics of neutrinos, axions and other weakly interacting particles Chicago: University of Chicago Press (1996)

[23] Miller Bertolami, M.M., Melendez, B.E., Altahus, L.G., Isern, J. Revisiting the axion bounds from the gali£; ctic white dwarf luminosity function JCAP 1410 (2014) 069

[24] Isern, J., Hernanz, M., García-Berro, E. Axion cooling of white dwarfs ApJ 392 (1992) L23

[25] Jaeckel, J., Ringwald, A. The Low-Energy Frontier of Particle Physic ARNPS 60 (2010) 405

[26] Ayala, A., Domï£¡nguez, I., Giannotti, M., Mirizzi, A., Straniero, O. Revisiting the bound on axion-photon coupling from globular cli£;sters. PRL 113 (2014) 191302

[27] Viaux, M., Catelan, M., Stetson, P.B., Raffelt, G., Redondo, J., Valcarce, A.A.R., Weiss, A. Neutrino and axion bounds from the gali£;ctic cli£;ster M5 (NGC 5904) PRL 111 (2013) 231301

[28] Córsico, A.H., et al. The rate of cooling of the pulsating white dwarf G1 17-B15A: a new asteroseismological inference of the axion mass MNRAS 424 (2012) 2792

[29] Córsico, A. H., et al. An independent li£;mit on the axion mass from the variable white dwarf star R548 JCAP 12 (2012) 10

[30] Isern, J., Garcï£¡a-Berro, E., Althaus, L.G., Cï£_rsico, A.,H. Axions and the pulsation periods of variable white dwarfs revisited A\&A $\mathbf{5 1 2}$ (2010) 86

[31] Giannotti, M., Irastorza, I., Redondo, J., Ringwald, A. Cool WISPs for stellar cooling excesses arXiv 1512.08108

[32] Kepler, S.O. Astr. Soc. Pacific C.S. 462 (2012) 322

[33] Mukadam, A. et al. Measuring the Evolutionary Rate of Cooling of ZZ Ceti ApJ 771 (2013) 17

[34] Friemann, J.A., Turner, M.S., Huterer, D. Dark energy and the accelerating universe ARAA 46 (2008) 385

[35] Bae, K.J., Baer, H., Chum, E.J. Mainly axion cold dark matter from natural supersymmetry JCAP 12 (2013) 028

[36] Ringwald, A., Saikawa, K. Accion dark-matter in the post-inflationary Peccei-Quinn symmetry breaking scenario arXive 1512.06436 (2016) 